\title{
BMJ Open Evaluation of a complex integrated, cross-sectoral psycho-oncological care program (isPO): a mixed-methods study protocol
}

Imke Jenniches, ${ }^{1}$ Clarissa Lemmen, ${ }^{2}$ Jan Christopher Cwik (i) ${ }^{3}$ Michael Kusch, ${ }^{4}$ Hildegard Labouvie, ${ }^{4}$ Nadine Scholten (D) , ${ }^{1}$ Alexander Gerlach, ${ }^{5}$ Stephanie Stock, ${ }^{2}$ Christina Samel, ${ }^{6}$ Anna Hagemeier, ${ }^{6}$ Martin Hellmich, ${ }^{6}$ Peter Haas, ${ }^{7}$ Michael Hallek, ${ }^{4}$ Holger Pfaff, ${ }^{1}$ Antje Dresen (D) ${ }^{1}$

To cite: Jenniches I, Lemmen C, Cwik JC, et al. Evaluation of a complex integrated, cross-sectoral psychooncological care program (isP0): a mixed-methods study protocol. BMJ Open 2020;10:e034141. doi:10.1136/ bmjopen-2019-034141

- Prepublication history for this paper is available online. To view these files, please visit the journal online (http://dx.doi. org/10.1136/bmjopen-2019034141).

Received 09 September 2019 Revised 17 December 2019 Accepted 20 December 2019

Check for updates

(C) Author(s) (or their employer(s)) 2020. Re-use permitted under CC BY-NC. No commercial re-use. See rights and permissions. Published by BMJ.

For numbered affiliations see end of article.

Correspondence to

Dr Antje Dresen;

antje.dresen@uk-koeln.de

\section{ABSTRACT}

Introduction International standards of care require the complete integration of psycho-oncological care into biomedical cancer treatment. The structured integrated, cross-sectoral psycho-oncological programme 'isPO' is aiming to ensure a provision of care in inpatient and outpatient settings according to a stepped-care approach. Up to now, psycho-oncological care is missing regulated and standardised processes to demonstrate the effectiveness. This study protocol describes the process and outcome evaluation that is conducted, along with the isPO study. The programme evaluation is aiming to proof effectiveness, explain potential discrepancies between expected and observed outcomes. Additionally, provide insight into the implementation process, as well as contextual factors that might promote or inhibit the dissemination and implementation of the stepped care programme will be gained. In addition to these measures, a cost-consequence analysis will provide further evidence aimed at integrating psycho-oncological care into primary healthcare.

Methods and analysis The evaluation concept is based on a tripartite strategy consisting of a prospective, formative and summative evaluation. To capture all determinants, a concurrent mixed-method design is applied comprising qualitative (interviews and focus groups) and quantitative (standardised questionnaires) surveys of patients and healthcare providers. In addition, analysis of the psycho-oncological care data (isP0 care data) and statutory health insurance claims data will be conducted. Primary and secondary data will complement one another (data linkage) to obtain a more comprehensive picture of the effectiveness and implementation of the complex intervention within the isP0 study.

Ethics and dissemination The study has been approved by the ethics committee of the Medical Faculty of the University of Cologne. For all collected data, the relevant national and European data protection regulations will be considered. All personal identifiers (eg, name, date of birth) will be pseudonymised. Dissemination strategies include annual reports as well as quality workshops for the organisations, the presentation of results in publications and on conferences, and public relations.
Strengths and limitations of this study

- The mixed-methods approach (qualitative and quantitative data) and the linking of primary and secondary data sources allow a multidimensional view on the quality and effectiveness of the psychooncological care programme.

- Results of the process evaluation are directly used to continuously improve the care programme.

- Regression discontinuity design allows estimation of the average treatment effect since a randomised controlled trial is not possible for ethical reasons in this specific setting.

- The cost consequence is of limited generalisability but is able to estimate a broader range of costs and consequences and help decision-makers structure their consideration of the different consequences of a decision.

- Risk of selection bias due to the quasi-experimental study design, in which patients are assigned to care-level groups on the basis of a cut-off value (validity of cut-off value will be analysed alongside with the primary outcome to ensure group equivalence).

Trial registration number DRKS00015326; Pre-results.

\section{INTRODUCTION}

There were 14.1 million new cancer cases and 32.6 million people living with cancer (within 5 years) in 2012 worldwide. In Germany, recent epidemiology studies showed an annual new cancer cases of about 480.000 cancer cases in $2014 .^{2}$ A cancer diagnosis is often associated with emotional distress as well as symptoms of anxiety and depression. ${ }^{3-5}$ Psychological well-being is increasingly seen as an important component of cancer care. In line with this, psycho-oncology is an area of multidisciplinary interest that deals with the psychological, social, behavioural and 
ethical aspects of cancer. ${ }^{6}$ This subspecialty addresses the two major psychological dimensions of cancer: the psychological responses of patients to cancer at all stages of the disease, and that of their families and caretakers; and the psychological, behavioural and social factors that may influence the disease process. Overall psychooncology aims to enable patients with cancer to cope with their illness and actively contribute to their medical treatment and follow-up care. ${ }^{6-8}$ Although the German national cancer plan ${ }^{9}$ as well national and international guidelines and standards of care ${ }^{10-12}$ call for the full integration of psycho-oncology into biomedical cancer treatment, essential structures, processes and financing for psycho-oncological treatment is not yet established. ${ }^{13-16}$ As funding is not uniformly regulated in Germany, the affordability of psycho-oncological treatment is a major challenge for private and statutory health insurances (SHIs). ${ }^{14-16}$

Systematic reviews and meta-analyses show that different psychotherapeutic and psychosocial intervention are associated with small-to-medium effects on quality of life and emotional distress. ${ }^{17-20}$ Effect size was positively correlated with high levels of distress at the starting point and the duration of intervention. ${ }^{17}$ However, most of the studies are restricted to female patients with breast cancer ${ }^{17} 21$ and do not comprise a predefined screening process of patients with cancer to deliver need-based psycho-oncological care in a steppedcare manner.

Therefore, the integrated cross-sectoral psychooncology (isPO) programme aims to develop, implement and evaluate a new stepped-care psychosocial and psychotherapeutic care programme for adult newly diagnosed patients with cancer. The German version of the widely accepted 'Hospital Anxiety and Depression Scale' (HADS) ${ }^{112223}$ and two self-developed and validated questionnaires (publication in preparation ${ }^{24}$ ), will be used as screening instruments to assess individual patient needs, that is, the assignment to a specific care level, and as an outcome measure. The regression discontinuity design (RDD) as a regression-based quasi-experimental approach is used to measure efficacy, reduction of anxiety and depression after 12 months of treatment via HADS questionnaire. Secondary outcomes are the quality of the care programme, including feasibility, patient satisfaction and perceived benefit.

Because an effectiveness study is limited in its ability to provide information regarding whether an intervention is successful (including the implementation process) ${ }^{26}$ and what are relevant costs and type of costs, the isPO study is accompanied by a complex process evaluation and a health economic analysis. This external evaluation aims to gain guidance on the quality and appropriateness of the psycho-oncological care programme and on implementation and dissemination strategies for complex health intervention. The evaluation will focus on implementation outcomes, such as acceptance, feasibility, appropriateness, effectiveness and costs, ${ }^{27}$ as well as patient-reported outcomes, such as quality of life and satisfaction with care.

In order to cover all relevant aspects, two frameworks are combined for the analysis: The Medical Research Council (MRC)-Framework for the evaluation of complex interventions $^{28}$ and the Consolidated Framework for Implementation Research (CFIR) ${ }^{29}$ The MRC guidance defines the function of process evaluation at different stages of development, evaluation and implementation of complex intervention and was therefore used to design the tripartite evaluation design, consisting of a prospective, formative and summative part. This framework gives equal attention to the implementation itself, the mechanism of impact (participant's responses and mechanisms of change) and the influence of contextual factors that may act as barriers and facilitators to implementation, dissemination and intervention effects. ${ }^{28}{ }^{30}$ For the systematic identification and evaluation of relevant context factors and potential barriers and facilitators, constructs related to an effective implementation of the CFIR are used. This framework captures the complexity of the implementation across the key aspects and provides a theoretically based coverage of the internal and external settings, the characteristics of the intervention and the people involved, and the process itself. This meta-theoretical framework can be used across all phases of implementation (preimplementation, during and postimplementation), ${ }^{29}{ }^{31}$ and has been shown to be effective for guiding successful implementation across numerous health domains, such as weight management and cancer screening. ${ }^{31}{ }^{32}$ Overall, both frameworks advocate a multilevel contextual perspective on the implementation and evaluation of interventions. The objective in using these two frameworks was to ensure that we took into account and addressed all the important factors based on well-validated constructs that are essential for the evaluation of a new health programme and its effective implementation.

\section{Main research questions}

- Does the structured 12-month psycho-oncological care programme reduce anxiety and depression in patients with cancer?

- How are quality of care, structures and processes perceived by patients and employees, and what is the experience of psychotherapists, social workers, oncoguides, nurses and physicians implementing isPO across settings?

- Which contextual factors and which baseline implementation constructs (CFIR) predict implementation success for each healthcare network?

- What effects does the isPO programme have on costs and use of SHI health services in comparison to standard care?

- Do the structures and contents of the healthcare programme provide a suitable basis for their integration in the standard healthcare system? 


\section{Secondary research questions}

- How do sociodemographic factors, comorbidities, the cancer entity or the form of medical treatment influence the effectiveness of psycho-oncological care?

- Which sociodemographic and healthcare-related risk factors predict the extent of psychological stress reactions on the initial diagnosis of cancer?

\section{METHODS AND ANALYSIS \\ Study design}

The project isPO aims to develop, implement and evaluate a new cross-sectoral form of psycho-oncological care. The study receives funding from the German Federal Joint Committee, a public legal entity comprising the four leading umbrella organisations of the self-governing German healthcare system. During the 4-year project (October 2017 to September 2021), up to 3484 outpatients and inpatients of full legal age $(\geq 18$ years $)$ with a primary diagnosis of cancer (International Statistical Classification of Diseases and Related Health Problems 10 - German Modification, ICD-10-GM, C00 - C97: Malignant neoplasms) will be included in the isPO programme. During the project period, patients will be treated for a period of 12 months from cancer diagnosis. Patients will be assigned to either the control group (HADS $\leq 14$; care levels 1 and 2) or the intervention group (HADS $\geq 15$; care level 3) depending on the anxiety and depression level on enrolment in the study. The control group will receive support of an onco-guide (level 1) or psychosocial support (level 2), whereas the intervention group receives psychotherapeutic treatment (level 3). Patients with a starting HADS \pm 2 around the cut-off will be evaluated for the treatment effect. This is based on the assumption that the distribution around the threshold is at random. ${ }^{33}$ The treatment effect (primary outcome) is analysed using the RDD for patients with initial HADS values between 13 and $16 .{ }^{34}$ The RDD represents a valid alternative to randomised controlled trial studies to estimate treatment effects. ${ }^{35}$ Anticipated treatment effects (reduction of HADS after 12 months of psycho-oncological treatment) are expected to lead to a discontinuity of the linear regression at the cut-off. In the course of the effectiveness measure, the preset cut-off value of the HADS will also be validated. In addition, further self-developed screening instruments are used to assess individual psychosocial risk factors and the cognitive-emotional response to cancer diagnosis and to assign patients to a certain level of care (level 1 or 2). Publication of the validation of these screening instruments is in preparation. ${ }^{24} 25$

In order to ensure the full integration of psychooncological treatment into clinical care, the psychooncological care is provided under supervision of the treating physician. The physicians, therefore, recommend their patients with cancer for the isPO programme. Depending on the needed level of care either oncoguides, trained former patients with cancer (level 1), social worker (level 2) or psychotherapists (level 3) will take over the treatment. Recruitment and care of the patients started in January 2019 and will continue until March 2021. (Publication of the detailed description of the psycho-oncological stepped-care concept of the isPO project is in preparation.)

The development and implementation of the isPO programme are based on the model of programme theory for health promotion programme. ${ }^{36}$ The process and outcome evaluation, carried out alongside the isPO care programme, applies a mixed-methods design based on several data sources, including health insurance claims data, clinical psychotherapeutic and psychosocial data (isPO care data) as well as qualitative and quantitative surveys of patients and healthcare providers. Both quantitative and qualitative data are collected in parallel and analysed separately. The results are then compared. In addition, there is an increasing need to assess the economic impact of psycho-oncology services on cancer in order to provide the necessary evidence to guide decision making. ${ }^{37}$ Not only the efficacy, but also the costeffectiveness of an intervention will be decisive, whether it will be transferred into standard care. Therefore, a health economic evaluation of isPO is necessary and also requested by the SHI. A cost-consequences analysis from the point of view of the German health insurance funds using SHI claims data is planned. This analysis examines costs and consequences without attempting to isolate a single consequence or aggregate consequences into a single measure. ${ }^{38}$ The health economic evaluation is financed by the University Hospital Cologne's own funds. Furthermore, fidelity, quality and feasibility will be monitored during the entire project using the clinical psychotherapeutic and psychosocial documentation (isPO care data), quarterly quality reviews of each network and quarterly internetwork and cross-network quality workshops.

\section{Setting}

The isPO study is conducted in four healthcare networks in North Rhine-Westphalia (Germany) each consisting of one hospital (rural, urban or university) with two or more certified oncological centres in collaboration with several resident physicians. (Contrary to hospitals, that provide basic medical cancer care, oncology centres bundle competences in the areas of treatment, cooperation and research. A certified oncological centre is a network of qualified and jointly certified, multidisciplinary and interdisciplinary, transsectoral and, if necessary, crosslocational facilities (hospitals, physicians, rehabilitation facilities) which best cover the various care areas for patients as far as possible. To meet the rapidly growing need for comprehensive, holistic, multidisciplinary and integrative oncological care of the population, these oncology competence centres have emerged). All four sites were selected based on predetermined criteria that are representative for the different healthcare providers in rural and urban regions. (Publication of the detailed description of the psycho-oncological stepped-care concept of the isPO project including the inclusion criteria for the networks is in preparation). For the 
process and outcome evaluation, all participating patients (approx. 3400) and healthcare providers (approx. 200), including nurses, psychotherapists, social workers and physicians, will be included in the quantitative study. For qualitative studies, participants will be selected based on the principal of 'purposeful sampling', a technique widely used in qualitative research for the identification and selection of information-rich cases for the most effective use of limited resources. ${ }^{39}$ This involves identifying and selecting individuals or groups of individuals that are especially knowledgeable about or experienced with a phenomenon of interest. ${ }^{39-41}$ The potential selection bias caused by this sampling method is offset by the mixedmethod design, which increases the credibility of results.

\section{Patient and public involvement}

The House of the Cancer Patient Support Associations of Germany (association of ten cancer support groups) as well as the German Cancer Society North RhineWestphalia are also applicants of the study. Both groups have been working on the concept, design and implementation since the planning of the study and give advice to the scientists regarding the outcome measures and research questions. They assess and represent the patient's perspective and will provide up-to-date information about the project status for the public on their homepages and events. In addition, all questionnaires were pretested with at least three former patients with cancer to ensure that the questionnaires met the needs of a patient in content, length and comprehensibility. The questionnaires were adapted as best as possible based on the patients' comments.

\section{Process and outcome evaluation design}

\section{Prospective, formative and summative evaluation}

Due to the high complexity of the isPO project, a multifaceted evaluation design is applied. For the current study, the MRC framework by Moore et $a l^{28}$ for process evaluation of complex interventions in combination with the CFIR constructs seems to be suitable. Therefore, a tripartite evaluation design will be applied comprising a prospective, formative and summative evaluation. Prospective and formative the concept, implementation and appropriateness will be evaluated on an ex ante and in-process basis. All results will be reported and promptly used to optimise the care programme and the implementation process. Finally, primary and secondary outcomes, as well as the transferability, will be evaluated summatively. By using this multidimensional mixed-method design, it will be possible to account for the different contextual factors influencing the implementation as well as patientrelated and patient-reported outcomes. Among others we will report implementation outcomes, such as acceptability, appropriateness, feasibility, fidelity, efficiency, effectiveness and satisfaction. ${ }^{27}$ In addition to patientrelated factors, such as age, comorbidities or sociodemographic factors, variables such as structural conditions, readiness and attitude towards change, knowledge-based and experience-based variables can play a role on the care provider's side. The planned data collection and the corresponding data sources as well as research questions are listed in table 1 .

\section{Quantitative data collection and analysis}

Within the scope of the study patients and healthcare provider, such as physicians, nurses, psychologists, social worker and onco-guides, are questioned at two points in time by means of a written (postal) survey. The aim of these surveys and the subsequent quantitative analysis is to determine the appropriateness and feasibility of the stepped-care programme. Essential aspects of the patient questionnaire are satisfaction with the psycho-oncological care, other social support and quality of life. The provider questionnaire focuses on the attitude towards the new psycho-oncological programme, intervention characteristics and personal as well as organisational barriers and facilitators, such as attitude towards change and corporate culture. ${ }^{29}$

In order to archive the highest possible response rate, the survey is conducted according to Dillman's 'Total Design Method'. ${ }^{42}$ To create the questionnaires and to import paper-based survey data, the data capturing software Teleform is used. Validated scales are analysed according to the coding manual. Psychometric analyses of factorial validity and reliability are carried out on the scales developed in-house.

\section{Qualitative data collection and analysis}

To enable patients to describe their experiences with the intervention, approximately 30 patients will be interviewed. In addition to the quantitative surveys, this qualitative analysis provides a deeper insight into the perceptions and opinions of the respondents. In addition, this method enables the identification of further factors that influence patient acceptance and perceived benefit. To meet the needs of vulnerable patients, such as patients with cancer, respondents can choose to participate in a single interview or focus group. The number of interviews will be adapted with respect to the saturation point. Participants will be approached through the data trustee of the project, who is the only one (other than the healthcare provider) with access to patient contact addresses for the data linkage and patient surveys. Through the targeted selection of interviewees, we take relevant criteria such as age, gender, assigned level of care and region into account. In addition, we will conduct approximately eight focus groups (six to eight persons each) with healthcare providers and interviews with each network coordinator (quality management representatives of the clinics), leading physicians and each leading psycho-oncologist of the four healthcare networks (four persons each). Interviews and focus groups will be guided by scientific rules, audio recorded, transcribed, pseudonymised and analysed based on content ${ }^{43}$ or documentary analyses. ${ }^{44}$

Overall, these qualitative analyses will provide in-depth understanding of the mechanisms of action, how context 


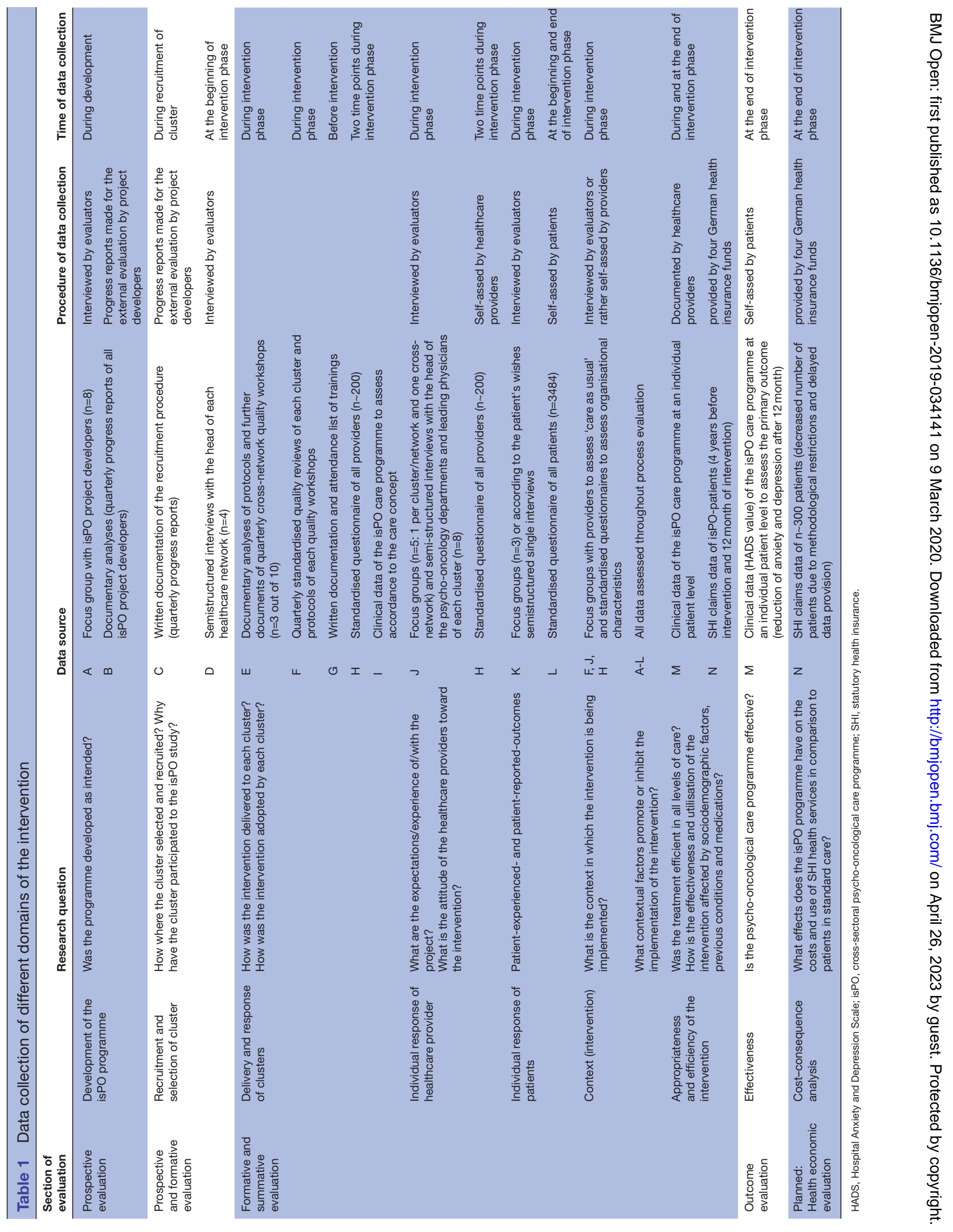


affects implementation or why those who carry out or receive the intervention engage as planned or not. It gives a greater insight into barriers and facilitators of the implementation process at the different sites as well as into the perception of different professions. In this way, we will collect data from all participants on key aspects of the process and combine these results with in-depth data from smaller samples.

Administrative data analysis and data linkage: $\mathrm{SHI}$ and clinical data In order to gain a greater insight into the impact of acute cancer treatment, health and medical history and comorbidities on the effectiveness of psycho-oncological care and the changing use of healthcare services during the care programme, the following data sets will be analysed: 1. SHI claims data of all recruited patients insured with one of the four largest German health insurance funds, covering around $70 \%$ of the statutory insured patients in Germany.

2. Clinical psychological and psychosocial data (isPO care data) from each recruited patient in each network (hospital).

3. Cancer registry data from each recruited patient in each network (hospital).

SHI claims data will be analysed at each care level within the framework of the stepped-care programme and at regional level (each network). In addition to patient characteristics, this hierarchical data set contains further information at the provider level. Data at the patient level include age, gender, insurance status, inpatient and outpatient treatment, diagnoses and drug prescriptions. At the provider level, it is possible to consider the attending physicians and hospitals at regional and medical specialist level. Hierarchical multilevel analysis allows us to consider the clustering of data at different levels in order to identify relevant influencing factors.

Clinical psychological and psychosocial data (isPO care data) of each recruited patient will provide information about the delivered care in each network and at each care level. This enables the isPO programme's range, fidelity and dose to be analysed.

A third data set (cancer registry data), containing the differentiated medical diagnosis and biomedical treatment, will provide additional information on the medical status of the patients included. This dataset will include additional clinical measures such as the tumour state, surgical procedures or the type of chemotherapy or radiotherapy. From the scope of cancer registry data, group differences are extracted, for example, in terms of age, entities or gender. The analyses will provide further insights into the influence of biomedical treatment or the severity of the disease on the effectiveness of psychooncological treatment.

All analyses follow the guidelines and recommendations of Good Practice of Secondary Data Analysis. ${ }^{45}$ As the data are not collected for scientific reasons but for reimbursement, resulting impact on the validity of the data is taken into account.
By linking the administrative data sets with the survey results, the perceived benefits and quality of life as well as sociodemographic data can be linked with the more general health status and the use of other health services. It also enables the identification of predictors of increased stress response after cancer diagnosis. In addition, multilevel analyses will take into account the health context in each network (hospital) and the relationship between the context and healthcare outcomes. ${ }^{46}$

\section{Health economic analysis}

A health economic analysis is conducted in form of a cost-consequences analysis from German SHI perspective. This economic evaluation is based on SHI claims data. The advantage of using routine data for evaluation is that cross-sectoral costs and the use of health services are taken into account. The result can help the decisionmakers to assess and compare the costs and use of health services between isPO patients and patients in standard care. The intervention group (isPO patient) is selected based on the is $\mathrm{PO}$ inclusion criteria, legal age ( $\geq 18$ years) and a primary diagnosis of cancer (ICD-10-GM C00-C97). However, due to the delayed availability of SHI claims data, not all isPO patients can be considered in the health economic analysis.

IsPO patients (intervention group) will be compared with patients in standard care (control group). The control group is generated based on SHI claims data using propensity score matching. ${ }^{47-49}$ Each isPO patient will be assigned to up to five patients from standard healthcare according to predefined criteria. Propensity score matching is a method to balance covariates observed in non-randomised studies between subjects in the control and intervention groups. The matching is carried out by the participating SHI funds, so that the data of the control group can then be transferred and analysed anonymously. Costs and use of inpatient and outpatient treatments, medicines, remedies and aids as well as periods of inability to work will be analysed. The economic evaluation examines differences in costs, use of health services and hospitalisation during 12 months of treatment in isPO compared with standard care. Costs and consequences of different outcomes are listed separately in a disaggregated format and the results are presented using descriptive statistics.

\section{Ethical conciderations}

For all analysed data relevant national and international data protection regulations will be respected. In accordance with national requirements and the principles of the Declaration of Helsinki, written informed consent will be obtained from all participants prior to enrolment and all study participants can revoke their consent without any negative consequences. The focus groups, as well as the interviews will be performed solely by trained researchers, trying to minimise the psychological burden of the patients as well as the healthcare providers. The survey will be performed pseudonymously. Personal 
identifiers will be only recorded to manage the responses to the questionnaire. Confidentiality will be maintained at all levels of data management. Cognitive pretest on the postal survey will be performed before dissemination in order to facilitate the answering of the questionnaire and reduce mental stress. The study information for patients is accompanied by a written consent, in which patients also give their written consent to the storage, processing and linking of all obtained data. This also includes the planned data linkage between the SHI claims data, the clinical psychological and psychosocial data (isPO care data) and the cancer registry data from each recruited patient in each network (hospital). All personal names are removed and all data records used are sufficiently coarsened to ensure pseudonymisation, especially with regard to data linkage. A complex approach to data protection, involving the use of three different pseudonyms (for the different data transmission channels) and the establishment of a project data trustee, who is not involved in data analysis, ensures the highest possible level of data protection in line with the requirements of the European General Data Protection Regulation. Up to the point of submission and although the study has received ethics approval, there was still no ethics vote available for the health economic analysis. Review of the health economics analysis by the ethical commission is currently in progress.

\section{Dissemination}

The isPO project partners have agreed on publication guidelines, a publication strategy and a publication plan. The publication strategy consists of the dissemination in scientific peer-reviewed journals and presentations at national and international academic conferences. Moreover, there is a strategy to ensure dissemination in popular science forums, such as research gate, and in public media. Professional exchange and patient participation will be a prominent task within in the project to gain the necessary dissemination and sustainability of the research findings. Therefore, a homepage was created to spread up to date news on the project progress. In addition, the House of the Cancer Patient Support Associations of Germany (association of 10 cancer support groups) as well as the German Cancer Society North Rhine-Westphalia will provide up-to-date information on their homepages and events.

The publication plan will evolve over time but includes the following planned scientific activities:

- Six Ph.D. theses at four different scientific institutes of the university of cologne.

- About 20 scientific articles in different peer-reviewed journals.

- Local seminars at the study sites.

- Information events of cancer self-help groups and the Cancer Society North Rhine-Westphalia.

- Special sessions planned at the 19th annual meeting of the PSO (Psycho-Oncology Committee of the German cancer Society) in 2020 and the German congress of Health Care Research (DKVF) 2020.

- Presentations at national and international conferences.

- Popular science presentations in national media and healthcare magazines.

\section{DISCUSSION}

IsPO aims to reduce symptoms of anxiety and depression in cancer inpatients and outpatients, to improve their psychosocial situation and to promote self-help. Different sectors of the German healthcare system and a wide range of scientific institutes cooperate to implement and evaluate a isPO more responsive to patients' needs. The evaluation of the new care programme will provide important evidence-based results from the perspective of patients and practitioners for cross-sectoral psychosocial and psycho-oncological care of patients with initial cancer diagnosis and opens the way for a transfer of the care programme into standard care. Cost-consequence analyses examine whether the care concept also favours the effective use of existing resources.

From a methodological point of view, the proposed project is highly innovative. It takes into account all relevant perspectives, enables new needs-based healthcare, has a prospective, multicentre and controlled design and offers a linkage of primary and secondary data from different health insurance funds for efficacy, effectiveness and cost analyses.

The aim of the project is to meet the requirements that make it possible to implement this new form of care in the standard care system in Germany. The care programme will combine approaches of cancer self-help (care level 1), psychosocial cancer counselling (care level 2) and psycho-oncological psychotherapy (care level 3) in a uniform care concept, operationalise them and integrate them into oncological care. It will provide a care management to regulate interdisciplinary and cross-sectoral cooperation in healthcare. Results of the process and outcome evaluation will provide insight into the quality and appropriateness of the care concept as well as the implementation process of a complex health innovation. In the long term, the programme should ensure care of patients with cancer from diagnosis to cure or palliative care and combine psychosocial and psychological care with further integrative oncology approaches, such as music and art therapy. In addition, the programme could also be suitable for the care of patients other than those suffering from oncological diseases.

A particular strength of the study is the participation of several SHI funds and a possible linkage of primary and secondary data. For the first time in Germany, this study, thus, offers an evidence-based basis for decision making on the integration of a new demand-oriented psycho-oncological care structure into standard healthcare. 
Author affiliations

${ }^{1}$ IMVR - Institute of Medical Sociology, Health Services Research and Rehabilitation Science (IMVR), University of Cologne, Cologne, Germany

${ }^{2}$ Institute of Health Economics and Clinical Epidemiology, University Hospital Cologne, Cologne, Germany

${ }^{3}$ Clinical Psychology and Psychotherapy, University of Cologne, Cologne, Germany

${ }^{4}$ Department of Internal Medicine, University Hospital Cologne, Cologne, Germany

${ }^{5}$ Department of Psychology, University of Cologne, Cologne, Germany

${ }^{6}$ Institute of Medical Statistics and Computational Biology, University of Cologne,

Cologne, Germany

${ }^{7}$ Department of Computer Science (Medical Informatics), University of Applied

Sciences and Arts Dortmund, Dortmund, Germany

Correction notice This article has been corrected since it was published. Affiliation for Christina Samel has been corrected.

Twitter Jan Christopher Cwik @Cwik_JC

Contributors As written in the main document, MaH, MK, MiH, AG, HP, NS, PH and SS are applicants of the funded study. IJ, AD, NS, JCC, CL, MH, CS, HL and MK designed the study. IJ drafted manuscript and incorporated the revisions between authors. $\mathrm{MH}, \mathrm{AH}, \mathrm{CS}, \mathrm{IJ}$ and $\mathrm{AD}$ drafted and finalised the concept of data protection for all patient related research data and data transfers involved as part of the ethics application. The final manuscript has been critically revised and approved by all authors.

Funding This study is funded by the Innovation Fund of the Federal Joint Committee, the G-BA (01NVF17022). The study has passed a peer-review selection process.

Competing interests None declared.

Patient consent for publication Not required.

Ethics approval The study was reviewed and has received ethics approval from the ethics committee of the Medical Faculty University hospital of Cologne and has been registered within the German Clinical Trial register (No. DRKS00015326).

Provenance and peer review Not commissioned; externally peer reviewed.

Open access This is an open access article distributed in accordance with the Creative Commons Attribution Non Commercial (CC BY-NC 4.0) license, which permits others to distribute, remix, adapt, build upon this work non-commercially, and license their derivative works on different terms, provided the original work is properly cited, appropriate credit is given, any changes made indicated, and the use is non-commercial. See: http://creativecommons.org/licenses/by-nc/4.0/.

\section{ORCID iDs}

Jan Christopher Cwik http://orcid.org/0000-0002-2290-353X

Nadine Scholten http://orcid.org/0000-0002-7793-7745

Antje Dresen http://orcid.org/0000-0001-7684-9391

\section{REFERENCES}

1 Torre LA, Bray F, Siegel RL, et al. Global cancer statistics, 2012. CA Cancer J Clin 2015;65:87-108.

2 Robert-Koch-Institut. Zentrum für Krebsregisterdaten. Krebs in Deutschland für 2013/2014, 2017. Available: https://www. krebsdaten.de/Krebs/DE/Content/Publikationen/Krebs_in Deutschland/kid 2017/krebs in deutschland 2017.pdf;jsessionid= F2403ECE5FDAC900A7D8783650BDCAF8.1_cid390?_blob= publicationFile

3 Linden W, Vodermaier A, Mackenzie R, et al. Anxiety and depression after cancer diagnosis: prevalence rates by cancer type, gender, and age. J Affect Disord 2012;141:343-51.

4 Zabora J, BrintzenhofeSzoc K, Curbow B, et al. The prevalence of psychological distress by cancer site. Psychooncology 2001;10:19-28.

5 Bussmann S, Vaganian L, Kusch M, et al. Angst und Emotionsregulation bei Krebspatienten. Psychotherapeut 2018;63:194-203.

6 Holland JC. Psycho-oncology. 3rd edn. New York: Oxford University Press, 2015.

7 Kusch M, Labouvie H, Hein-Nau B, et al. Berlin/Heidelberg. Springer, 2013.

8 Weis J, Schumacher A, Blettner G, et al. Psychoonkologie. Onkologe 2007;13:185-94.
9 Bundesministerium für Gesundheit (BMG). Nationaler Krebsplan: Handlungsfelder, Ziele, Umsetzungsempfehlungen und Ergebnisse, 2012. Available: https://www.bundesgesundheitsministerium. de/fileadmin/Dateien/5 Publikationen/Praevention/Broschueren/ Broschuere_Nationaler_Krebsplan.pdf

10 Institute of Medicine (IOM). Cancer care for the whole patient: meeting psychosocial health needs. Washington, DC: National Academies Press, 2008.

11 Leitlinienprogramm Onkologie (DKG, DKH, AWMF). Psychoonkologische Diagnostik, Beratung und Behandlung von erwachsenen Krebspatienten: Langfassung version 2014.

12 Holland J, Watson M, Dunn J. The IPOS new international standard of quality cancer care: integrating the psychosocial domain into routine care. Psychooncology 2011;20:677-80.

13 Jacobsen PB, Lee M. Integrating psychosocial care into routine cancer care. Cancer Control 2015;22:442-9.

14 Singer S, Dieng S, Wesselmann S. Psycho-oncological care in certified cancer centres--a nationwide analysis in Germany. Psychooncology 2013;22:1435-7.

15 Singer S, Hornemann B, Bruns G, et al. Organisation Der psychoonkologischen Versorgung. Forum Fam Plan West Hemisph 2016;31:124-9.

16 Heckl U, Singer S, Wickert M, et al. Aktuelle Versorgungsstrukturen in Der Psychoonkologie. Nervenheilkunde 2011;30:124-30.

17 Faller H, Schuler M, Richard M, et al. Effects of psycho-oncologic interventions on emotional distress and quality of life in adult patients with cancer: systematic review and meta-analysis. J Clin Oncol 2013;31:782-93.

18 Osborn RL, Demoncada AC, Feuerstein M. Psychosocial interventions for depression, anxiety, and quality of life in cancer survivors: meta-analyses. Int J Psychiatry Med 2006;36:13-34.

19 Sheard T, Maguire P. The effect of psychological interventions on anxiety and depression in cancer patients: results of two metaanalyses. Br J Cancer 1999;80:1770-80.

20 Zimmermann T, Heinrichs N, Baucom DH. "Does one size fit all?" moderators in psychosocial interventions for breast cancer patients: a meta-analysis. Ann Behav Med 2007;34:225-39.

21 Kerr A, Ross E, Jacques G, et al. The sociology of cancer: a decade of research. Sociol Health IIIn 2018;40:552-76.

22 Ibbotson T, Maguire P, Selby P, et al. Screening for anxiety and depression in cancer patients: the effects of disease and treatment. Eur J Cancer 1994;30A:37-40

23 Jacobsen PB, Donovan KA, Trask PC, et al. Screening for psychologic distress in ambulatory cancer patients. Cancer 2005;103:1494-502.

24 Kusch M, Cwik JC, Bussmann S, et al. Fragebogen Zur kognitivemotionalen Auseinandersetzung MIT Krebs (KEA-K. Köln: CIO Köln, 2017.

25 Cwik JC, Bussmann S, Vaganian L, et al. Fragebogen zu Psychosozialen Risiken (PSR. Köln: Universität zu Köln, 2018.

26 Oakley A, Strange V, Bonell C, et al. Process evaluation in randomised controlled trials of complex interventions. BMJ 2006;332:413-6.

27 Proctor E, Silmere H, Raghavan R, et al. Outcomes for implementation research: conceptual distinctions, measurement challenges, and research agenda. Adm Policy Ment Health 2011;38:65-76.

28 Moore GF, Audrey S, Barker M, et al. Process evaluation of complex interventions: medical Research Council guidance. BMJ 2015;350:h1258.

29 Damschroder LJ, Aron DC, Keith RE, et al. Fostering implementation of health services research findings into practice: a consolidated framework for advancing implementation science. Implement Sci 2009;4:50.

30 Craig P, Dieppe P, Macintyre S, et al. Developing and evaluating complex interventions: the new medical Research Council guidance. BMJ 2008;337:a1655.

31 Damschroder LJ, Lowery JC. Evaluation of a large-scale weight management program using the consolidated framework for implementation research (CFIR). Implement Sci 2013;8:51.

32 Liang S, Kegler MC, Cotter M, et al. Integrating evidence-based practices for increasing cancer screenings in safety net health systems: a multiple case study using the consolidated framework for implementation research. Implement Sci 2016;11:109.

33 Venkataramani AS, Bor J, Jena AB. Regression discontinuity designs in healthcare research. BMJ 2016;352:i1216.

34 Moscoe E, Bor J, Bärnighausen T. Regression discontinuity designs are underutilized in medicine, epidemiology, and public health: a review of current and best practice. J Clin Epidemiol 2015;68:132-43.

35 Maas IL, Nolte S, Walter OB, et al. The regression discontinuity design showed to be a valid alternative to a randomized 
controlled trial for estimating treatment effects. J Clin Epidemiol 2017;82:94-102.

36 Issel LM. Health program planning and evaluation: a practical, systematic approach for community health. 3rd edn. Burlington Mass.: Jones and Bartlett Learning, 2014.

37 Tuffaha H, El-Saifi N, Chambers S, et al. New challenges in psychooncology: economic evaluation of psychosocial services in cancer: challenges and best practice recommendations. Psychooncology 2019;28:3-10.

38 Brazier J, Ratcliffe J, Salomon JA, et al. Measuring and valuing health benefits for economic evaluation. 2nd edn. New York: Oxford: Oxford Univ. Pr, 2017.

39 Patton MQ. Qualitative research and evaluation methods. 3rd edn. Thousand Oaks: Sage, 2002.

40 Palinkas LA, Horwitz SM, Green CA, et al. Purposeful sampling for qualitative data collection and analysis in mixed method implementation research. Adm Policy Ment Health 2015;42:533-44.

41 Creswell JW, Plano Clark VL. Designing and conducting mixed methods research. Los Angeles, London, New Delhi, Singapore, Washington DC, Melbourne: Sage, 2018.

42 Dillman DA. Mail and telephone surveys: the total design method. New York: Wiley \& Sons, 1978.
43 Mayring P. Qualitative Inhaltsanalyse: Grundlagen und Techniken. 12th edn. Weinheim: Beltz, 2015.

44 Bohnsack R. Gruppendiskussionsverfahren und dokumentarische Methode. in: Schaeffer D, Müller-Mundt G, EDS. qualitative Gesundheits- und Pflegeforschung. Bern: Huber 2002:305-25.

45 Swart E, Gothe H, Geyer S, et al. Gute praxis Sekundärdatenanalyse (GPs): Leitlinien und Empfehlungen. Gesundheitswesen 2015;77:120-6.

46 Ansmann L, Kuhr K, Kowalski C. Mehrebenenanalysen in Der organisationsbezogenen Versorgungsforschung - Nutzen, Voraussetzungen und Durchführung. Gesundheitswesen 2017;79:203-9.

47 Austin PC. An introduction to propensity score methods for reducing the effects of confounding in observational studies. Multivariate Behav Res 2011;46:399-424.

48 Austin PC. Optimal caliper widths for propensity-score matching when estimating differences in means and differences in proportions in observational studies. Pharm Stat 2011;10:150-61.

49 Rosenbaum PR, Rubin DB. Constructing a control group using multivariate matched sampling methods that incorporate the propensity score. The American Statistician 2012;39:33-8. 